emetica and many another in the autumn as they "went into the fields and down the lane" with strict orders to avoid Mr MacGregor's garden.

To Beatrix Potter's coloured pictures, many of which have the authentic touch of genius, have been added almost an equal number of excellent plates by R. B. Davis and E. C. Large. Around these illustrations, and ten half-tone photographic plates the reproduction of which leaves much to be desired, Dr Findlay has woven a text. The first six chapters form a readable introduction to the larger fungi which the amateur will read with profit and which will give him added pleasure as he strolls through the woods in autumn casually trying to match the fungi he finds with the pictures in the book. The seventh to sixteenth chapters are devoted largely to the descriptions of individual species. The deseriptions are simple and reflect Dr Findlay's profound knowledge of fungi. They are, however, clearly not intended for the more serious student and references to microscopic features and spore measurements have been avoided.

Perhaps the weakest parts of the book are the halfdozen black and white figures. For example, Fig. 3 gives a very poor and misleading picture of the beautiful microscopic structure of the hymenium in toadstools. It is easy, however, to criticize a work of this kind, but on the whole it is to be welcomed more on artistic and nostalgic rather than on scientific grounds, for all the time in appreciating Beatrix Potter's artistic skill in depicting a toadstool we can almost see Mr Jeremy Fisher or Squirrel Nutkin peeping round the corner. It is a pity that the book is so expensive that fow individuals will be able to afford it.

C. T. INGOLD

\section{AN EXPANDING TECHNOLOGY}

\section{Progress in Microbiological Techniques}

Edited by C. H. Collins. Pp. ix +231 . (London : Butterworth and Co. (Publishers), Ltd., 1967.) $55 s$.

THIs collection of eleven papers reports contemporary developments in some of the more fascinating and sophisticated techniques used by microbiologists and those working in related fields. The editor of this volume, C. H. Collins, has selected a broad range of techniques for discussion, including several which are of direct relevance to the applied microbiologist.

Clear, critical accounts are given of complement fixation, serological analysis of fungi and actinomycetes, fluorescent antibody methods and the freeze-drying of micro-organisms. Some specific needs of industrial and public health laboratories are considered in chapters on the microbiological assay of vitamins and amino-acids, standardization of vaccines, evaluation of yeasts and moulds for brewing and antibiotic production, phage typing of staphylococci and the examination of water supplies. This last chapter contains a particularly good appraisal of membrane filtration procedures and comments on the respective merits of currently available membranes. Most of the contributors direct their readers to sources of materials demanded by the methods described, whether they be items of equipment, reference cultures or serological reagents. One of the expressed hopes for this book is that it will be useful to microbiologists who may be required occasionally to apply any of the techniques discussed to their own peculiar problems. It is essential, therefore, to have a rapid and reliable reference to appropriate suppliers and this exigency is met almost to the full. Progress in Microbiological Techniques will also make valuable reading for teaching purposes and the chapter on the analysis of the bacterial cell gathers together an abundance of useful practical information. This book can be recommended strongly and any adverse criticism is slight and minor. One hopes that the producers of the present volume will be eneouraged to think of it as the forerunner of others that will continue to survey, at a reasonable price, the development and application of new and specialist techniques.

Alan T. BUll

\section{LEARNING BY PROBLEM SOLVING}

\section{Problems and Solutions in Mathematical Physics}

By Y. Choquet-Bruhat. Translated by C. Peltzer. Translation edited by J. J. Brandstatter. (Holden-Day Series in Mathematical Physics.) Pp. ix + 314. (San Francisco, London and Amsterdam : Holden-Day, Inc., 1967.) $\$ 10.00$.

ThIs attractive book is aimed at introducing students of theoretical physics, applied mathematics, and engineering, to some of the modern mathematical methods and concepts. It is not a book of rules of what to do, and it will appeal more to those people, both undergraduate and graduate, who care about what they are doing. The treatment is more rigorous than will appeal to some readers, though there is no undue emphasis on achieving the utmost generality ; the work is essentially as simple as is compatible with ensuring the right answers.

There are 125 separate problems, many split into about five related parts, with full solutions. One of the first things to strike one is the great variety of topics covered. A list of the eight main section headings will give a rough idea of its scope : (1) Linear mappings. Operations on matrices. (2) Proper values and proper vectors. Reduction of matrices. (3) Scalar product and norm. Hermitian operators. (4) Vector calculus. Multiple integrals. (5) Function spaces and operators. (6) Series expansions of functions. (7) Differential equations. (8) Partial differential equations. Some especially interesting features are the use of differential forms in parts of section $\mathbf{4}$, for example in a problem involving covariant derivatives and symmetries of the Riemann-Christoffel tensor, and the use elsewhere of the theory of distributions.

I found the problems interesting and well chosen. Nearly all make a useful point or illustrate some important principle. The solutions are helpful and seem generally, though not universally, correct. Some of the English translation reads rather awkwardly, but there are few places where the meaning is not immediately clear. On certain pages there are quite a lot of trivial misprintssixteen on page 217. In some cases these defects, or incomplete explanations or definitions, make it difficult to interpret the question without first looking at the solution. The matter referred to in some footnote references. for example, " . . . (see Problem Paris 1957)", on page 248 , could not be found. Although the table of contents lists every problem by name, a subject index would still have been useful.

Even with the defects mentioned here, this seems to me to be a useful addition to the problem books on mathematical physics, and provides an enjoyable and interesting way of learning an important branch of the subject.

A. Weinmann

\section{CHARGE CARRIERS IN ORGANIC SOLIDS}

\section{Organic Semiconductors}

By Felix Gutmann and Lawrence E. Lyons. (Wiley Series on the Science and Technology of Materials.) Pp. xvii + 858. (New York and London: John Wiley and Sons, 1967.) $224 s$.

MODERN interest in the semiconducting properties of organic crystals started about 20 years ago, with measurements on phthalocyanine crystals, first as powders, then as single erystals. The work was chiefly initiated by 